NIST Technical Note 2030

\title{
A Calibration of Timing Accuracy in the NIST Cyber-Physical Systems Testbed
}

\author{
Marc Weiss \\ Ya-Shian Li-Baboud \\ Dhananjay Anand \\ Kevin G. Brady Jr. \\ Paul Boynton \\ Cuong Nguyen \\ Martin Burns \\ Avi Gopstein
}

This publication is available free of charge from:

https://doi.org/10.6028/NIST.TN.2030

National Institute of 


\title{
A Calibration of Timing Accuracy in the NIST Cyber-Physical Systems Testbed
}

\author{
Marc Weiss \\ Dhananjay Anand \\ Paul Boynton \\ Cuong Nguyen \\ Martin Burns \\ Avi Gopstein \\ Office of Smart Grid and Cyber-Physical Systems \\ Engineering Laboratory \\ Ya-Shian Li-Baboud \\ Kevin G. Brady Jr. \\ Software Systems Division \\ Information Technology Laboratory
}

This publication is available free of charge from:

https://doi.org/10.6028/NIST.TN.2030

December 2018

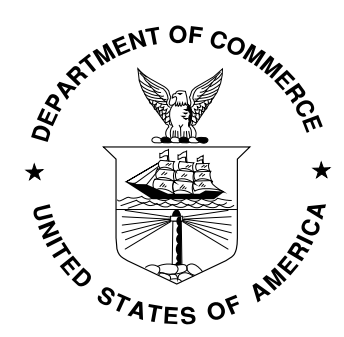

U.S. Department of Commerce Wilbur L. Ross, Jr., Secretary

National Institute of Standards and Technology Walter Copan, NIST Director and Undersecretary of Commerce for Standards and Technology 
Certain commercial entities, equipment, or materials may be identified in this document in order to describe an experimental procedure or concept adequately. Such identification is not intended to imply recommendation or endorsement by the National Institute of Standards and Technology, nor is it intended to imply that the entities, materials, or equipment are necessarily the best available for the purpose.

National Institute of Standards and Technology Technical Note 2030 Natl. Inst. Stand. Technol. Tech. Note 2030, 30 pages (December 2018) CODEN: NTNOEF

This publication is available free of charge from:

https://doi.org/10.6028/NIST.TN.2030 


\begin{abstract}
We propose a general methodology for assessing the time accuracy and uncertainties, and report results from a project to calibrate timing in the NIST Cyber-Physical System (CPS) and Smart Grid Testbeds. We measured clock synchronization accuracy and stability as well as latencies for potential experiments in the testbeds. We determined calibrations of GPS receivers to UTC(NIST) with an uncertainty of 16 ns. However, an anomaly occurred coincident with a power shutdown, which resulted in a total uncertainty of receiver calibrations against UTC(NIST) of 100 ns. Synchronization at testbed locations relative to an IEEE 1588 Precision Time Protocol (PTP) grandmaster was found to have a max offset of 36 ns \pm 6 ns one sigma from the grandmaster through two transparent clocks (TCs). Finally, we measured the time error relative to the grandmaster of an embedded device attached to a switch without PTP support with a mean offset of $50 \mu \mathrm{s} \pm 10 \mu \mathrm{s}$, and at $8 \mathrm{~ms} \pm 500 \mu \mathrm{s}$ for timestamping at the general-purpose input/output (GPIO). We report the methodology used, as well as some of the challenges encountered and solutions developed in the process.
\end{abstract}

\title{
Key words
}

Timing characterization, Cyber-Physical Systems, Internet of Things, testbeds 


\section{Table of Contents}

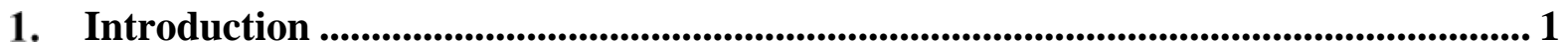

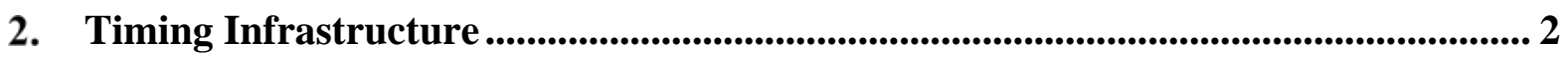

2.1. Establishing a UTC reference........................................................................ 2

2.2. Uncertainty of round-trip Rubidium calibration ....................................................... 5

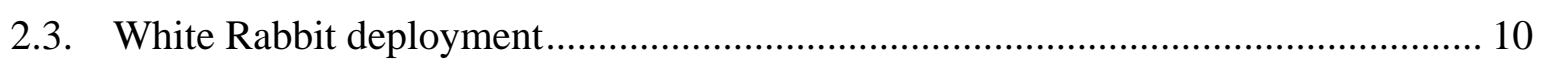

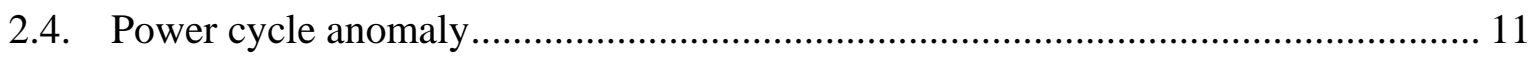

2.5. White Rabbit Grandmaster Characterization ...................................................... 12

2.6. White Rabbit Slave Characterization ................................................................... 13

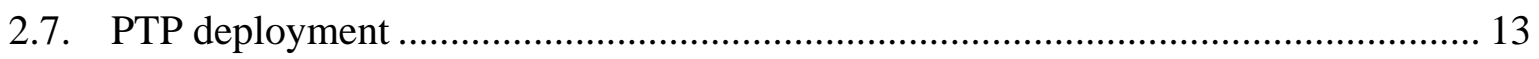

3. Timing Characterization Methodology and Results ......................................................... 14

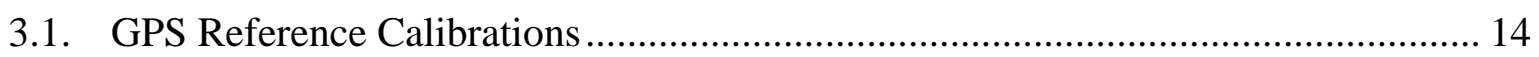

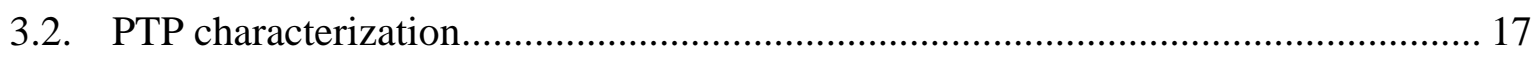

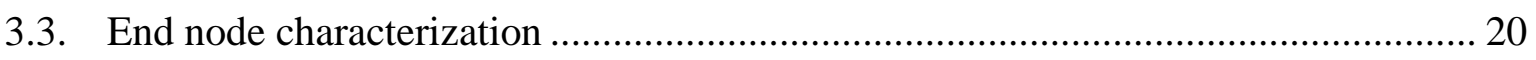

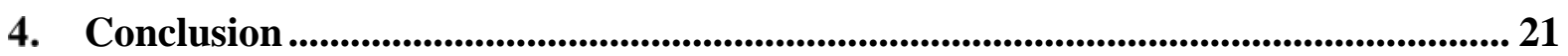

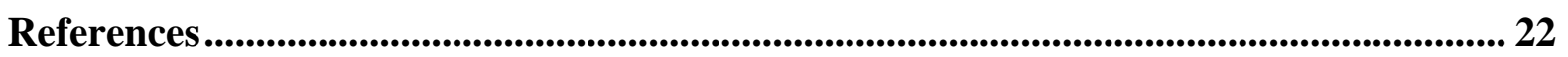

\section{List of Tables}

Table 1. Uncertainty against UTC(NIST) For the Rb Clock Calibrations............................... 10

Table 2. White Rabbit slave calibration against the EL DA................................................. 13

Table 3. Testbed GPS Receiver Offsets from UTC(NIST) via Rb Calibrations against the

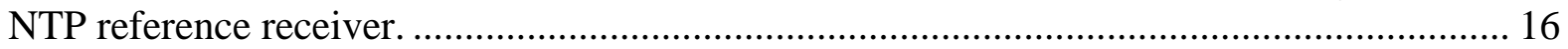

Table 4. GPS Antenna Cable Measurements ............................................................................ 17

Table 5. Network Device Time Uncertainty Contribution Using PTP Peer-to-Peer Layer 218

Table 6. Network Device Time Uncertainty Contribution Using PTP End-to-End Layer 3.19

\section{List of Figures}

Fig. 1. Common view time transfer, showing cancellation of the ephemeris delay for various baselines between national timing laboratories. ...................................................................... 3

Fig. 2. Differential calibration for common view time transfer. ................................................ 3

Fig. 3. Schematic diagram of timing reference devices....................................................... 4

Fig. 4. Free running Rb against GPS Rb Disciplined Oscillator (DO).................................. 6

Fig. 5. Data from Fig. 4 after removing a mean 1st difference of time, and the remaining

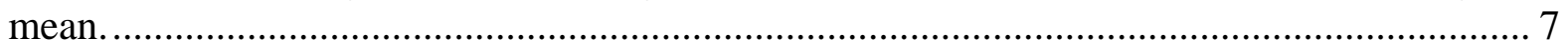

Fig. 6. Residuals of the free-running Rb against the CCF reference after removing a mean 1st difference of time, and the remaining mean. 
Fig. 7. Time Deviation (TDEV) and Time Total Deviation of the data from Fig. 4, with respect to GPS-disciplined Rb (left) and using the data of Fig. 6 with respect to the Cs (right).

Fig. 8. Two days of 1 PPS data of the WR Slave device in the testbed measuring GPS1, with adjacent $100 \mathrm{~s}$ intervals averaged, and mean removed. This suggests an uncertainty of $10 \mathrm{~ns}$ for about a two minute measurement of a receiver against the Rb during a calibration trip.... 9 Fig. 9. Efficient TDEV of GPS2 monitored by the WR Slave in the testbed from almost one day of data. The maximum deviation is about $5 \mathrm{~ns}$ at $300 \mathrm{~s}$.

Fig. 10. Offset between EL DA, the source for the WR GM, and the WR slave in a loop back configuration, with 88 ns mean removed.......................................................................... 11

Fig. 11. Smart Grid CPS testbed network............................................................................ 14

Fig. 12. Transparent clock characterization topology. The lines in red denote the measured signals or packets. The offsets are measured by the time interval counter and the time stamp and packet delays are measured by the network synchronization tester..

Fig. 13. Time offset distribution between the Cs reference source (via the WR network) and the PTP slave via 2 TCs configured as PTP P2P Layer 2. 19 Fig. 14. Time offset distribution between the Cs reference source (via the WR network) and the PTP slave via 2 TCs configured as PTP E2E Layer 3 with a PTP GM emulation........... 20

Fig. 15. TDEV comparison of testbed time reference distribution methods.......................... 20

Fig 16. Test topology with software-based PTP timestamping and a non-PTP switch........... 21

Fig. 17. Test setup for estimating the aggregate timestamp error of a 'typical' IoT node synchronized to an NTP server. 


\section{Introduction}

Timing is a fundamental property in the metrology of Cyber-Physical Systems (CPS), from large-scale deployments such as the power grid, to smaller scale Internet of Things (IoT) networks such as body area networks. All require aspects of timing to enable sensor fusion, ordering of events, and timeliness, ensuring that measurements and information remain meaningful in dynamically evolving environments.

The National Institute of Standards and Technology (NIST) has constructed a Smart Grid and Cyber-Physical Systems (CPS) testbed to study behaviors of composed simulated, emulated, and actual hardware components of systems and systems of systems. This testbed has been developed to house experiments designed and implemented by a multi-disciplinary group of researchers at NIST and their collaborators around the globe. It comprises several adjacent modules in one building on the NIST Gaithersburg campus.

NIST anticipates mounting devices and embedded components in its testbed. The network in this testbed is a simple tree and branch structure of switches which support IEEE 1588 Precision Time Protocol (PTP) for accurate time propagation. Devices such as desktop computers, virtual machines, embedded computing systems and actual fielded IoT and Smart Grid devices are attached to the leaf switches in the testbed. This managed time distribution model facilitates minimum uncertainty of synchronized time throughout the testbed.

To understand the underlying timing uncertainties in these components in any given experiment, it is essential to understand the basic behavior of the testbed with regard to time. Time and timing uncertainty can propagate to measurement uncertainty and therefore information and prediction uncertainty. Many systems require synchronization with respect to Coordinated Universal Time (UTC), particularly wide-area based systems or systems operated by multiple vendors. UTC is a post-processed time scale. Any real-time realization of UTC is only a prediction of what the correct time will be some time later, when it is defined. A lab that participates in the generation of International Atomic Time (TAI) and UTC produces its own real-time prediction of UTC, denoted UTC(lab), where "lab" is a 3- or 4-letter acronym for the timing lab. Hence our goal has been to characterize the uncertainty against a reference source traceable to UTC(NIST) [1][2]. To this end, we describe the infrastructure and calibrations we established to create a means to measure and monitor timing uncertainty and stability.

The contribution of this work is the integration of measurement technologies and methodologies to enable real-time end-to-end measurement traceability from UTC(NIST) to commercially available GPS receivers, down to device timing performance, including general purpose input/output (GPIO) timestamping latency and variation. The understanding of temporal uncertainties whether it is synchronization or latency variation, can enhance future distributed measurement and analysis algorithms. Better knowledge and confidence of the temporal validity and uncertainty of the data can therefore be achieved. Temporal validity is application dependent in the testbed, and current industry clock synchronization, frequency and latency requirements vary from hundreds of nanoseconds to seconds [3]. 


\section{Timing Infrastructure}

\subsection{Establishing a UTC reference}

UTC(NIST) is generated at the NIST labs in Boulder, Colorado; however, an established traceable source of UTC(NIST) exists on the Gaithersburg campus, specifically for the NIST Network Time Protocol (NTP) servers that are located there. The offset from UTC(NIST) on the Gaithersburg campus in the Central Computing Facility (CCF) is based upon common view time transfer with Boulder, Colorado [4]. Common view time transfer cancels many of the errors from GPS. Fig. 1 shows the cancellation of satellite ephemeris errors in common view between NIST, Boulder, and other timing labs around the world.

To get time from any receiver, the delay through the system from the antenna to the PPS output must be calibrated. For common view time transfer, only the differential delay is required, between the user's receiver and the reference receiver. For a differential calibration in general, the receiver to be calibrated is measured against the reference receiver with both using a common clock, UTC(NIST) in our case, and a short baseline. Each receiver has a unique antenna with known coordinates, separated by a short enough baseline that they see the same ionosphere and troposphere, as illustrated in Fig. 2. Then measurements are taken against UTC(NIST) by each receiver and against the same satellites at the same time, hence the phrase common view. With some averaging this gives the differential delay between the receiver-antenna pairs. In principle, it is best to include the operational antenna cable for both receivers in this calibration. In practice, it is sometimes too difficult to remove a cable from its permanent installation to do a differential calibration measurement. In this case, the cables for the device under test can be measured. With an uncertainty of a few nanoseconds, it is possible to measure the delay in both the antenna cable used for calibration and in the operational cable. This is how the reference receiver for NTP in the CCF was calibrated to provide UTC(NIST). 


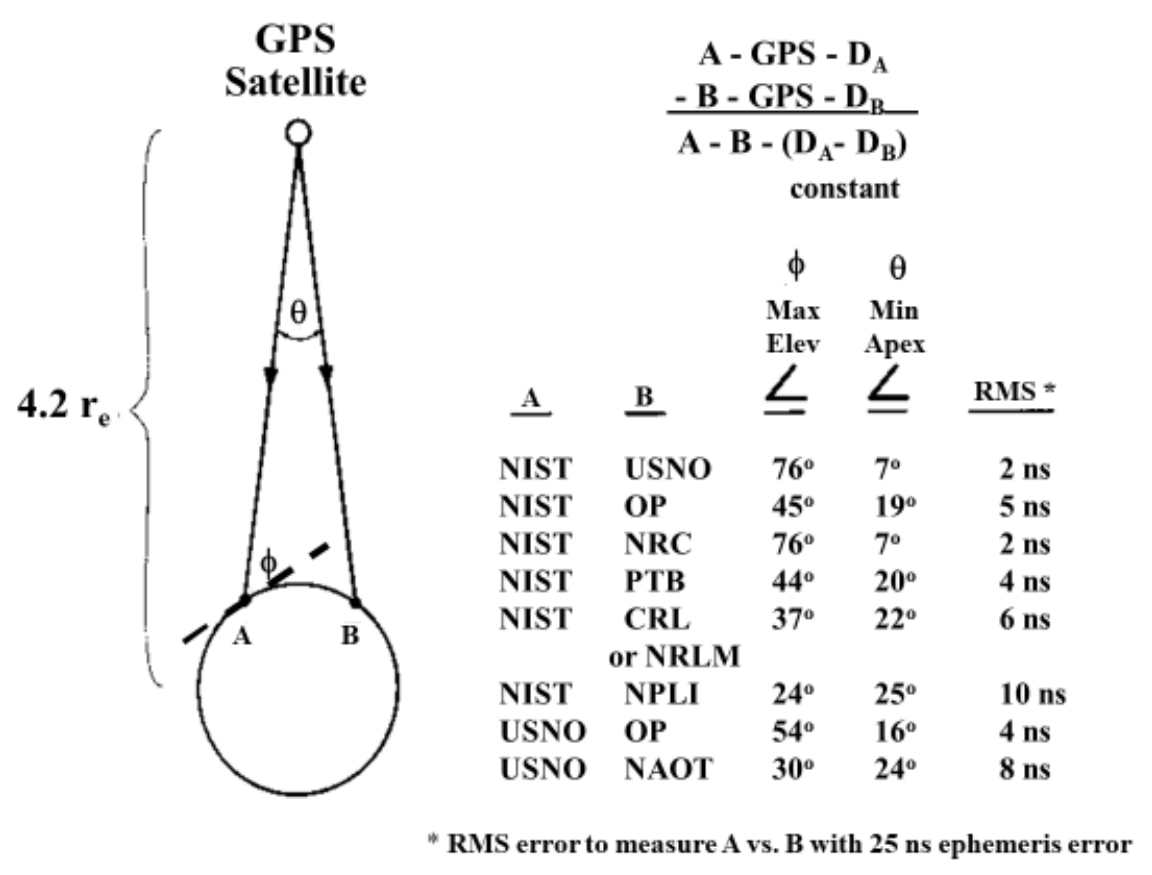

Fig. 1. Common view time transfer, showing cancellation of the ephemeris delay for various baselines between national timing laboratories.

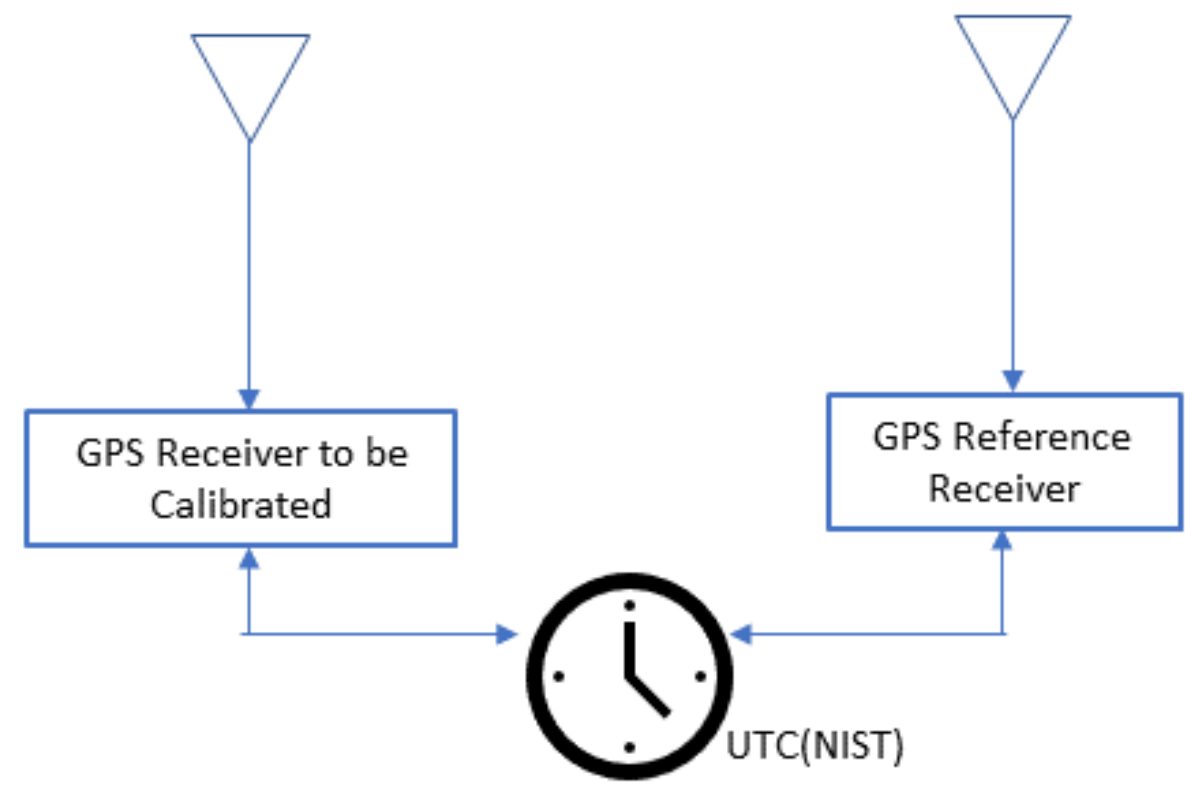

Fig. 2. Differential calibration for common view time transfer.

Several GPS receiver references were established to provide UTC within the testbed, and the CCF source was used to calibrate the testbed GPS receivers. Once calibrated, the testbed 
receivers could also serve as a PTP and NTP grandmaster (GM) for devices on the testbed. The UTC source for the NTP server in the CCF includes a Cesium (Cs) reference with its PPS output accessed through a distribution amplifier (NTP DA), which is the UTC reference plane for that system. The testbed is several buildings away from the CCF, approximately $450 \mathrm{~m}$ apart.

The PPS output of the NTP DA is connected to a pulse distribution amplifier (DA) owned by the NIST Engineering Lab (EL). We refer to this new DA as the EL DA, and it serves to keep a level of separation between the NTP UTC and the testbed apparatus. We measured the difference of the NTP DA minus the EL DA to be 21 ns. In this paper, measurements are stated without uncertainties when the uncertainties are under 1 ns. The signals from the DAs were generated by Complementary Metal Oxide Semiconductor (CMOS) logic, and triggered at $1.3 \mathrm{~V}$ at $50 \Omega$.

The time and frequency signals from the NTP's Cs reference were provided as reference signals for a White Rabbit (WR) [5] Grandmaster (GM). The purpose of the WR was to distribute the time reference signal from the NTP Cs in the testbed. This would allow long term comparison of the time from a GPS receiver in the testbed with UTC(NIST) via the NTP reference system. Initially, as shown later, this allows us to reduce the uncertainty of the receiver calibrations. In addition, the WR system allows tracking of any wander in the calibrated GPS signals used as the local time reference within the testbed. We configured GPS receivers as well as a WR slave in the testbed area. Both the EL DA and the WR GM were co-located in the CCF with the NTP UTC reference and the NTP DA. Fig. 3.

Schematic diagram of timing reference devices.gives the schematic diagram of the reference devices.

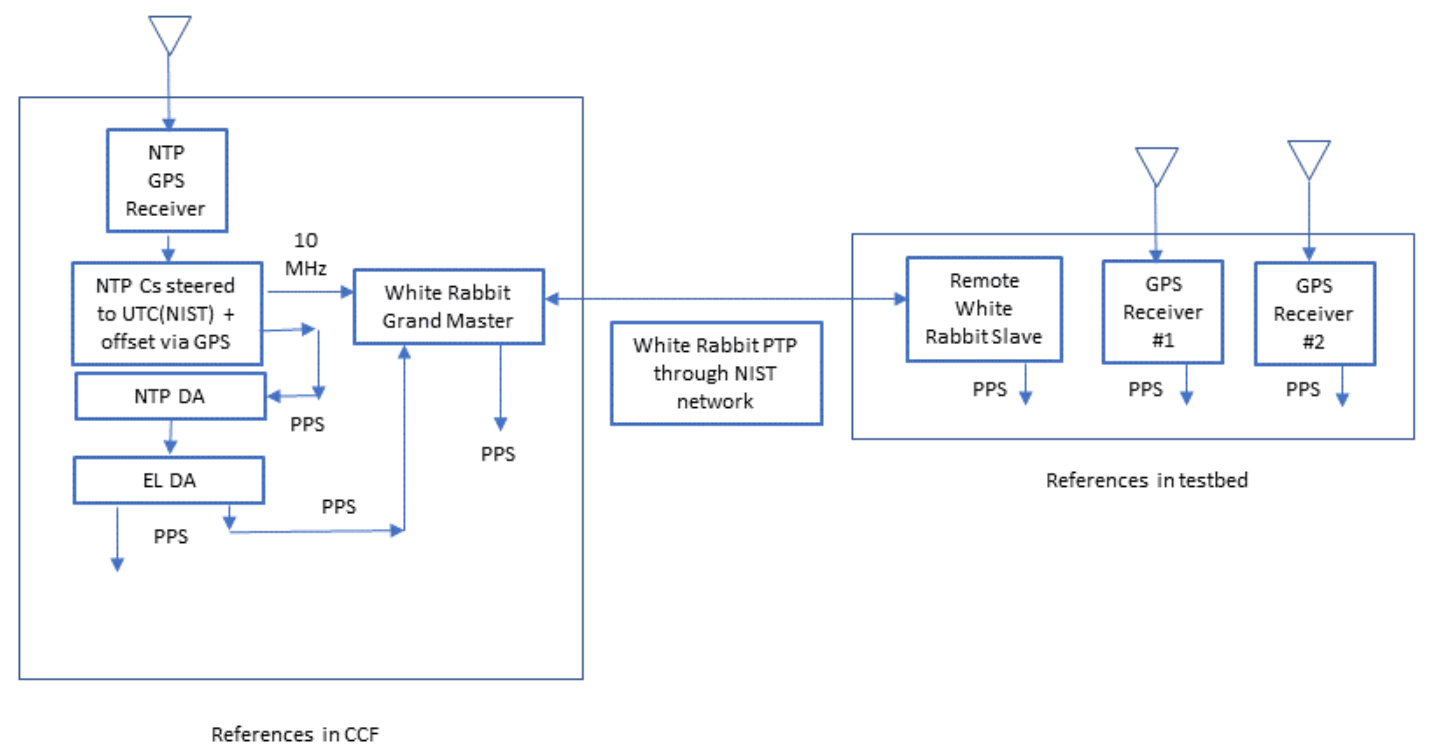

Fig. 3. Schematic diagram of timing reference devices. 


\subsection{Uncertainty of round-trip Rubidium calibration}

GPS receivers were established in the testbed reference lab. The accuracy of time from a receiver is unknown without a calibration of the delay through the antenna, the antenna cable, and the receiver, plus any external cables delivering 1 PPS. All GPS receivers were calibrated against the NTP UTC reference in NIST Gaithersburg using a portable Rubidium $(\mathrm{Rb})$ clock to transfer time in physical round-trip measurements. The use of Rb portable clocks has been an established method for measuring the time delay between different locations [6]. The portable Rb trips calibrated both the GPS receivers and the remote WR slave device co-located with the receivers at the testbed lab. The sequence for calibration was as follows.

1. Lock Rb to EL DA for over 1.5 hours, then disconnect.

2. Measure the free running $\mathrm{Rb}$ against EL DA.

3. Transport Rb clock to area of device(s) under test (DUTs), and measure free running $\mathrm{Rb}$ against DUTs.

4. Transport Rb clock back to EL DA and measure the free running Rb.

Thus, the Rb clock was measured at the beginning and end of the calibration trip against our reference plane in the CCF. Measurements were made using a Time Interval Counter (TIC) to measure the time interval between a PPS from the Rb and one from the EL DA. After measuring the $\mathrm{Rb}$ against the EL DA at the beginning and end of the round-trip, we determine a slope from the mean first difference of these values. This slope, plus the initial $\mathrm{Rb}$ offset, is then used to determine the value of the EL DA and then UTC(NIST) against the DUTs.

Between those times, the Rb was free-running, with unknown performance. The expected performance during free-run was determined from measuring free-running performance multiple times against GPS-disciplined references. Before we had access to the CCF Cs reference, we used a $\mathrm{Rb}$ disciplined oscillator (DO) in a different lab. The instability of the $\mathrm{Rb}$ while it is free-running during the round-trip is a significant source of the calibration error. To characterize the magnitude of this error, we measured the behavior of the Rb clock while free-running in various circumstances. Fig. 4. Free running Rb against GPS Rb Disciplined Oscillator (DO)., Fig. 5 and Fig. 6 show the behavior of the free running Rb when measured against a GPS disciplined Rb oscillator. Fig. 4 is the data as taken after locking showing the residual rate offset. Fig. 5 shows the same data after removing a mean $1^{\text {st }}$ difference and the residual mean from those data. This is approximately what we do during the $\mathrm{Rb}$ calibration trips where we approximate a linear behavior using the last and first measurements against the EL DA in the CCF. That is, what you see in Fig. 5 is what the free-running $\mathrm{Rb}$ would do in this instance during the calibration trip when it is not being measured, assuming the reference were perfect. The peak-to-peak variation for the full interval of almost $0.7 \mathrm{~d}$ or about $17 \mathrm{~h}$ is about $15 \mathrm{~ns}$. But our Rb clock trips were typically closer in length to $2-3 \mathrm{~h}$, or about $0.1 \mathrm{~d}$. There are periods in Fig. 5 where there are sharp changes in slope, i.e. frequency steps during $0.1 \mathrm{~d}$ periods. However, it is not clear in these data how much is due to the free-running $\mathrm{Rb}$ oscillator that we later use for calibration and the reference clock in this case, which is also a Rb oscillator, though locked to a GPS receiver. Fig. 6 gives the Time Deviation (TDEV) [7] and Time Total Deviation [8] of the data in Fig. 4 and Fig. 5. The deviation of these data at 2 to 3 hours is about 3 ns. When measured against the Cs. clock in the CCF, we generally see the peak-to-peak variation of the 
free-running $\mathrm{Rb}$ during an interval of under 5 hours after locking was about 2 ns, as shown in Fig. 7. Conservatively, considering the possibility of unobserved frequency steps, we estimate $5 \mathrm{~ns}$ for the round-trip calibration uncertainty due to the $\mathrm{Rb}$ instability.

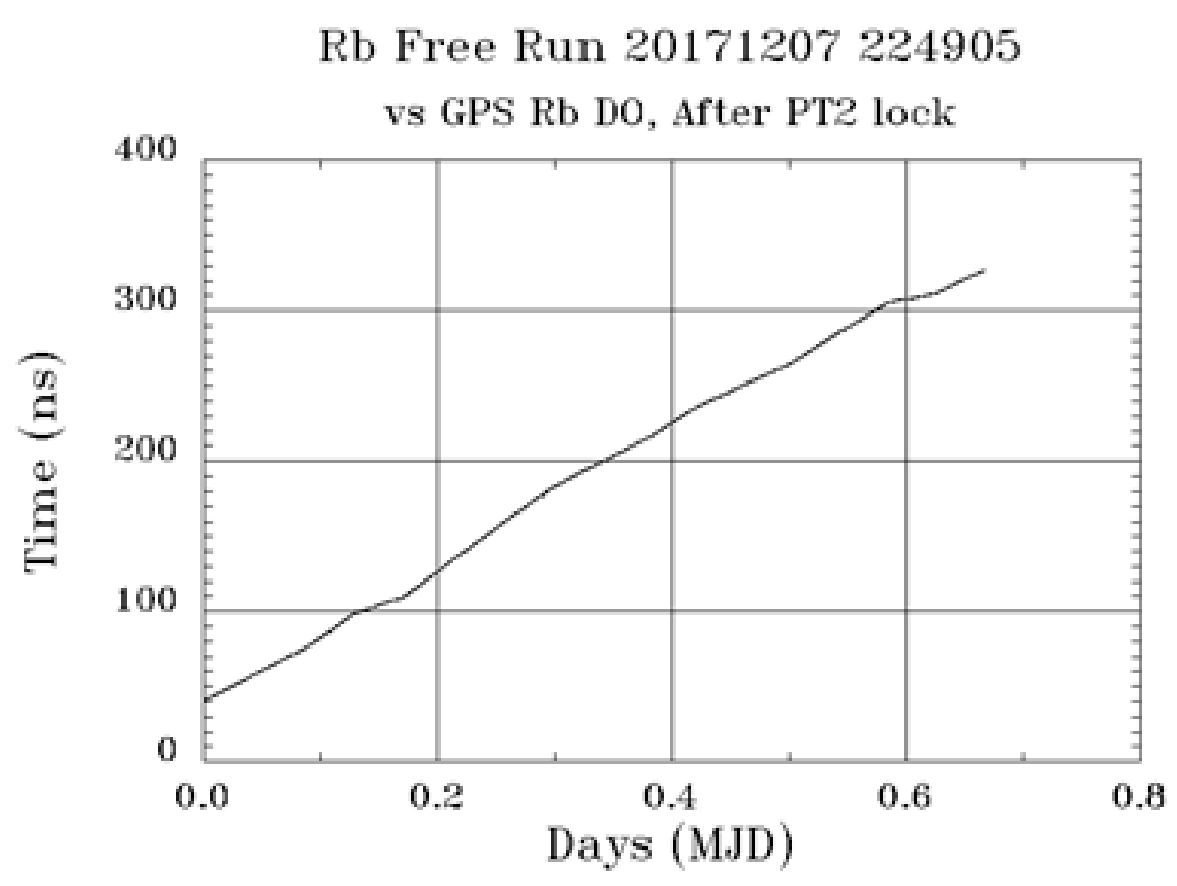

Fig. 4. Free running Rb against GPS Rb Disciplined Oscillator (DO). 
Rb Free Run 20171207224905

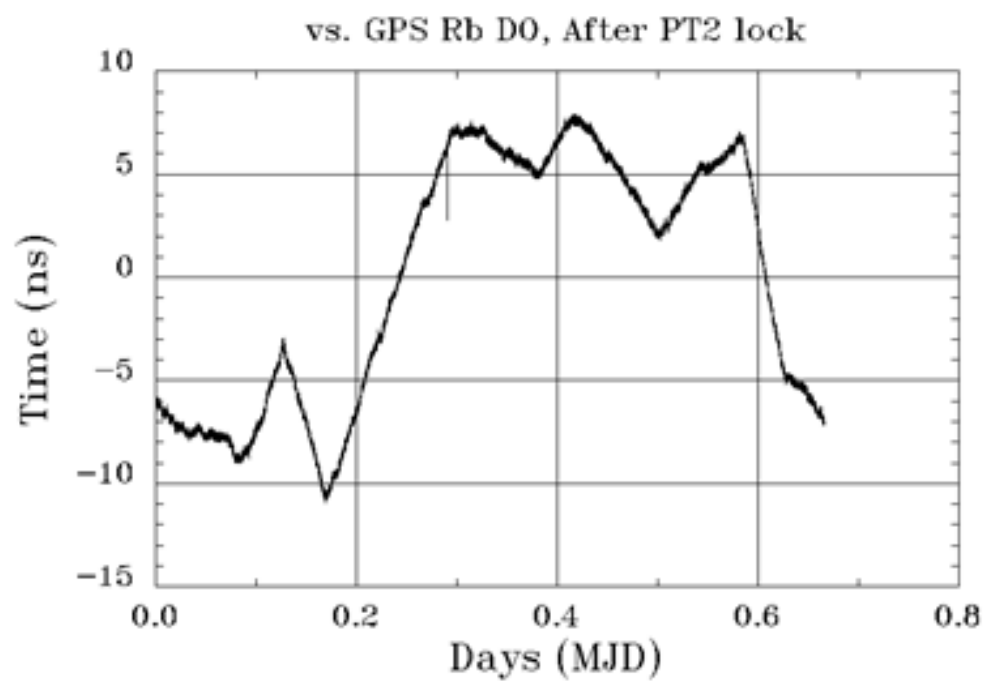

Fig. 5. Data from Fig. 4. Free running Rb against GPS Rb Disciplined Oscillator (DO). after removing a mean 1st difference of time, and the remaining mean.

Rb Free Run vs CCF Cs

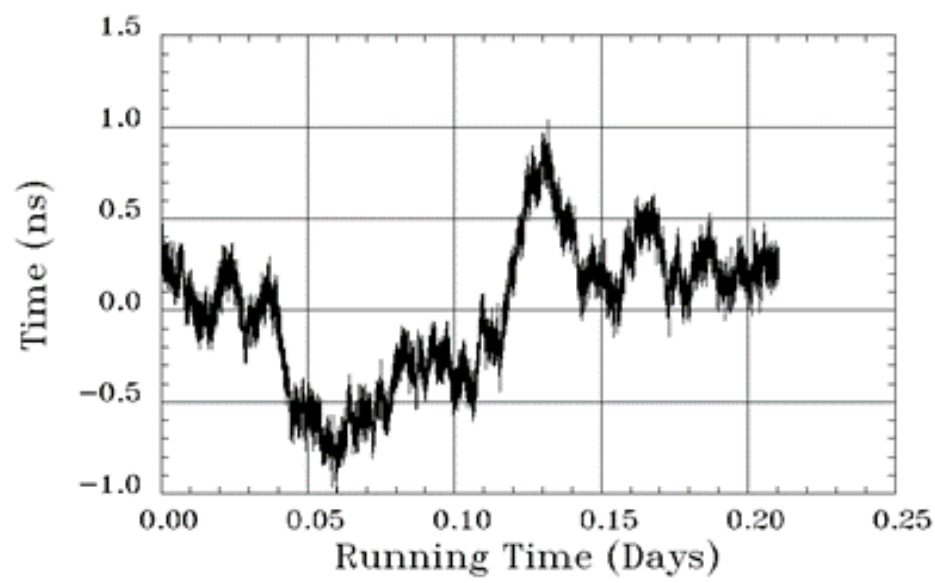

Fig. 6. Residuals of the free-running $\mathrm{Rb}$ against the CCF reference after removing a mean $1 \mathrm{st}$ difference of time, and the remaining mean. 

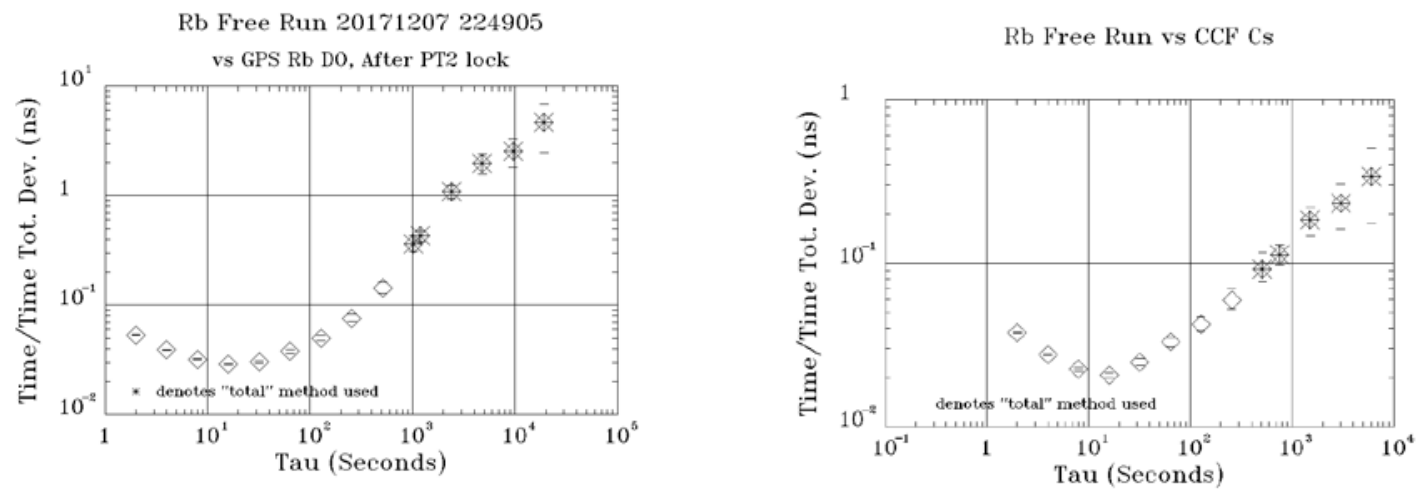

Fig. 7. Time Deviation (TDEV) and Time Total Deviation of the data from Fig. 4, with respect to GPS-disciplined Rb (left) and using the data of Fig. 6 with respect to the Cs (right).

Measurements of the Rb against the DUTs were generally limited in time to about 2 minutes to minimize the free-running time of the $\mathrm{Rb}$. This became the dominant uncertainty for the calibrations, as the instability of various receivers we estimate conservatively as up to 10 ns over intervals this short. Fig. 8 shows a two-day measurement of one of the GPS receivers, GPS1, against the WR Slave device in the testbed, and with adjacent intervals of $100 \mathrm{~s}$ averaged to one point. We can see that peak deviations in this plot are up to 8 ns. Fig. 9 shows the TDEV values of a different receiver, GPS2, with a maximum of about 5 ns at 300 s of averaging. These data were from a monitoring of GPS2 by the WR Slave in the testbed over almost one day. Here the efficient algorithm was used [7], which increases the speed of computation when there are more equivalent degrees of freedom than needed for confidence. As we argue in section 2.6, the long-term instability of the WR Slave against the EL DA was under 2 ns. These two plots show examples of the data that form the basis of estimating 10 ns uncertainty for our measurements of GPS receiver time from a 1 PPS signal with lengths under 2-3 minutes. 


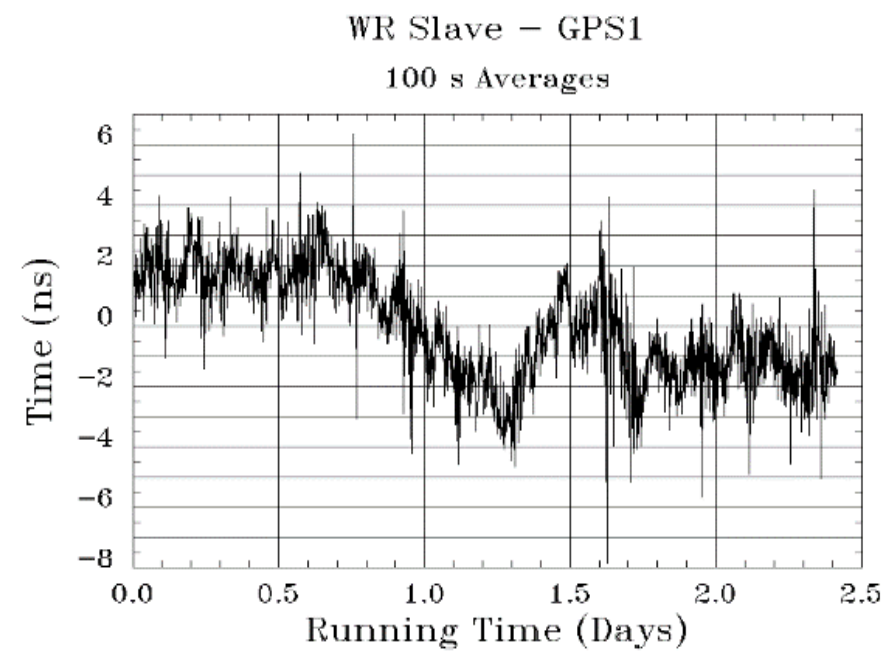

Fig. 8. Two days of 1 PPS data of the WR Slave device in the testbed measuring GPS1, with adjacent $100 \mathrm{~s}$ intervals averaged, and mean removed. This suggests an uncertainty of $10 \mathrm{~ns}$ for about a two minute measurement of a receiver against the $\mathrm{Rb}$ during a calibration trip.

WR Slave - GPS2

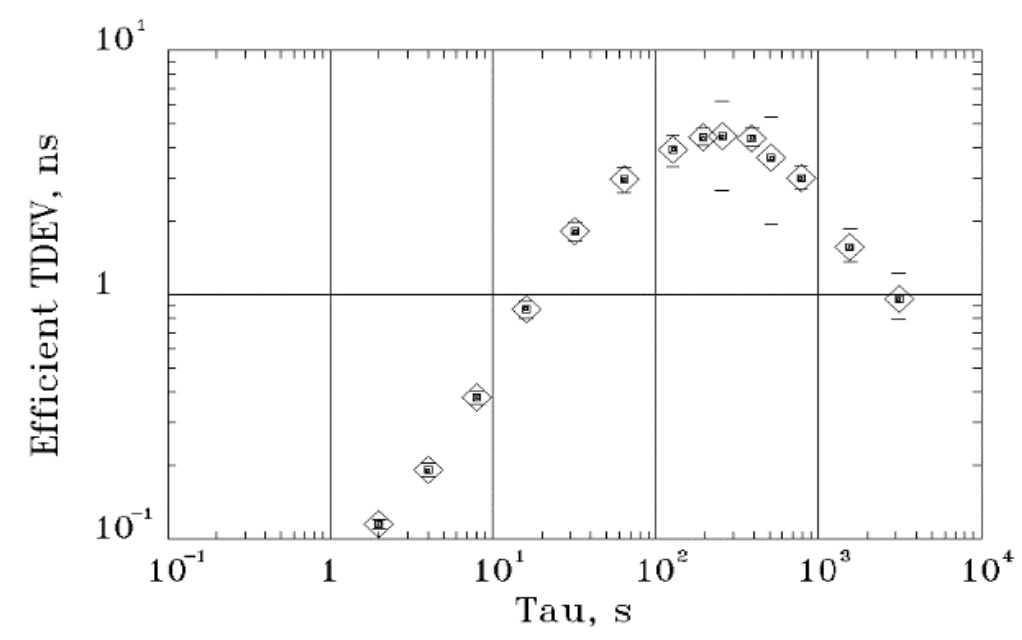

Fig. 9. Efficient TDEV of GPS2 monitored by the WR Slave in the testbed from almost one day of data. The maximum deviation is about $5 \mathrm{~ns}$ at $300 \mathrm{~s}$.

Thus from the data in Fig. 8 and Fig. 9 we take an uncertainty of 10 ns for the peak error in the measurement of a receiver against the $\mathrm{Rb}$ for 2 minutes, and, combining the square root of the sum of the squares of these two uncertainties, we have:

$$
\Delta C_{\text {total }}=\sqrt{\Delta R c v r^{2}+\Delta R b^{2}}
$$


where the receiver uncertainty, $\Delta R c v r$, is $10 \mathrm{~ns}$ and the $\mathrm{Rb}$ uncertainty, $\Delta R b$, is $5 \mathrm{~ns}$, giving a total uncertainty against the CCF Cs reference plane, $\Delta C_{\text {total }}$, of $11 \mathrm{~ns}$ for the calibrations.

The uncertainty against UTC(NIST) must, in addition, include the uncertainty of the common-view time transfer, and of the differential receiver calibration used to measure the GPS receiver in the CCF against the NIST primary receiver in Boulder. Uncertainties for common view are discussed in references [9],[10], and we conservatively estimate 8 ns for this element. Finally, differential calibration uncertainties and the variation of a receiver delay with time is studied in [9],[10]. We conservatively estimate an uncertainty of 8 ns for the differential receiver delay calibration of the receiver used in the NTP reference as calibrated against the NIST primary receiver in Boulder. Then we combine these as the root sum square, as in (1),

$$
\Delta U_{\text {total }}=\sqrt{\Delta R c v r^{2}+\Delta R b^{2}+\Delta C v u^{2}+\Delta D C a l^{2}},
$$

where we have added to the terms under the radical in Eq. (1) $\Delta C v u$, the common-view time transfer uncertainty of $8 \mathrm{~ns}$, and $\Delta D C a l$, the differential receiver calibration uncertainty of 8 ns, yielding a total uncertainty for the calibration against UTC, $\Delta U_{\text {total }}$, of $16 \mathrm{ns,} \mathrm{well} \mathrm{below}$ our goal of $100 \mathrm{~ns}$ for the delivering UTC to endpoints in the testbed. This is presented in Table 1. Uncertainty against UTC(NIST) For the Rb Clock Calibrations.

Table 1. Uncertainty against UTC(NIST) For the Rb Clock Calibrations

\begin{tabular}{|c|c|c|}
\hline Source of uncertainty & Uncertainty ns & Support in this paper \\
\hline Free-running Rb Clock & 5 & Fig. 2 \\
\hline $\begin{array}{l}\text { Variations in PPS from } \\
\text { receivers, beyond } \mathrm{Rb} \\
\text { measurements }\end{array}$ & 10 & Fig. 8 and Fig. 9 \\
\hline $\begin{array}{c}\text { Common-view time } \\
\text { transfer }\end{array}$ & 8 & References [9], [10] \\
\hline $\begin{array}{l}\text { Differential receiver } \\
\text { delay calibration }\end{array}$ & 8 & References[9], [10] \\
\hline Total uncertainty & 16 & Equation (2) \\
\hline
\end{tabular}

\subsection{White Rabbit (WR) deployment}

The WR system was deployed to extend the NTP UTC traceable Cs reference into the testbed to enable remote long-term measurements of the GPS-derived time reference. For the WR system to do this, both the WR GM and the WR Slave in the testbed needed to be calibrated against the NTP DA. As illustrated in Fig. 1, the $10 \mathrm{MHz}$ and 1 PPS signals from the NTP Cs reference were connected as input to the WR Switch operating in Grandmaster mode (WR GM). When turned on, the WR GM uses the 1 PPS to lock on a zero-crossing of the $10 \mathrm{MHz}$ signal. Thereafter, this $10 \mathrm{MHz}$ phase from the Cs is the time reference for the WR GM. Calibration of the WR GM is required both because the offset of the phase of the $10 \mathrm{MHz}$ signal from the 1 PPS is unknown, and because the delay between this $10 \mathrm{MHz}$ phase and the internal reference time in the WR GM is also unknown. Calibration of the WR GM was done simply by measuring the 1 PPS out from the GM against the 1 PPS from the EL DA, since the WR GM was co-located with the equipment in the CCF. This measurement was repeated often and is discussed below. 
Calibration was required for the WR Slave device in the testbed, or any other WR Slave device, because there is no way of knowing the accuracy of the WR system without independently measuring it. In principle, the White Rabbit system as developed and deployed at Conseil Européen pour la Recherche Nucléaire (CERN) [5] has been shown to have sub-ns accuracy. In practice, however, this performance depends on several system functions that may not be performing optimally, such as reciprocity of the two-way delay in the network, and calibration of the WR nodes. Calibration of a WR Slave against the EL DA required the use of the portable Rb clock, the same method used to calibrate the GPS receivers in the testbed. These round-trip portable $\mathrm{Rb}$ measurements were used to calibrate the accuracy of the WR slave in the testbed against the EL DA, and hence against UTC(NIST).

The WR slave at the testbed locked on the GM using the White Rabbit technology [5] across the NIST optical fiber backbone via ten patches. The long-term stability of the slave against the master was verified using a loop-back from the reference to the testbed slave and back to a second slave node co-located with the WR GM. The 1 PPS outputs of the co-located WR slave minus the EL DA were measured. The peak-peak value over a weekend test was under 0.5 ns, as shown in Fig. 10. This plot, as an example, justifies estimating the stability of the reference time of the Slave against the WR GM to be less than 1 ns. As discussed in section 2.6, repeated traveling Rb clock calibrations of the WR Slave device support a conservative uncertainty of less than 2 ns.

WR Loopback

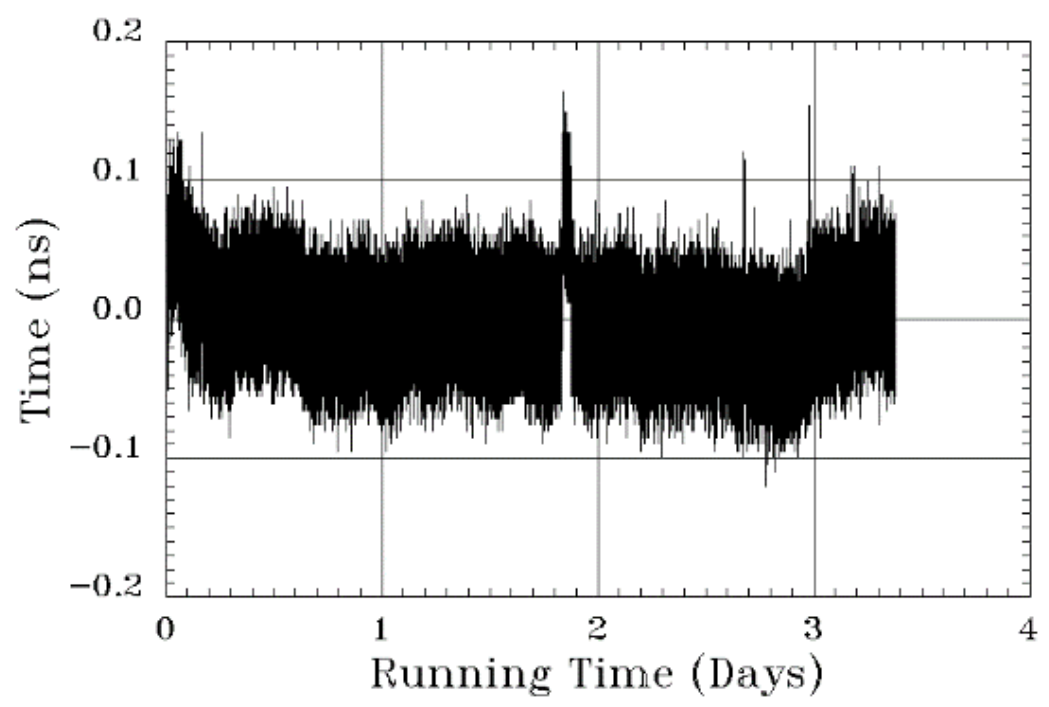

Fig. 10. Offset between EL DA, the source for the WR GM, and the WR slave in a loop back configuration, with 88 ns mean removed.

\subsection{Power cycle anomaly}

During the measurement process, a power cycle of the CCF was coincident with a step change in the calibration results. The CCF is expected to be powered continually with multiple uninterruptible power backup systems. As an extremely unusual event, there was a requirement to bring down power in the entire CCF over a weekend. The power was brought 
down from Friday April 13 through Sunday April 15. During subsequent sections of this paper, we will refer to changes in calibration as Pre-Power Cycle and Post-Power Cycle. There were two measured anomalies. First, the calibration of GPS receivers in the testbed relative to the NTP reference receivers in the CCF moved by of order 100 ns, considering the \pm 10 ns uncertainty. Because of this, we assign an uncertainty of $100 \mathrm{~ns}$ to the calibration of receivers in the testbed against UTC(NIST). For resolution, we had a receiver calibrated directly against UTC(NIST) in Boulder, Colorado. We will have another new receiver calibrated as well, once it is purchased. If these two new calibrations agree we will reduce our calibration uncertainty of GPS receivers in the testbed against UTC(NIST). The second anomaly we found was that the calibration of the WR GM against the EL DA moved by 13 ns. We will explore in a subsequent paper the causes of these anomalies.

\subsection{White Rabbit Grandmaster Characterization}

As discussed above, the 1 PPS output from the WR GM was measured against the 1 PPS from the EL DA using a time-interval counter in order to calibrate the internal reference time of the WR GM. Before the power cycle in the CCF this value, (EL DA)-(WR GM), was consistently $88 \mathrm{~ns}$, rounded to the nearest ns, over many measurements. After the power cycle, this value was consistently $75 \mathrm{~ns}$, rounded to $1 \mathrm{~ns}$. The value of NTP DA minus EL DA was consistently $21 \mathrm{~ns}$ from before to after the power cycle. So, the 13 ns change seems to be due to either a change in the relation between the Cs PPS and $10 \mathrm{MHz}$ phase, or due to a change in the WR GM's generation of its $1 \mathrm{PPS}$, relative to the $10 \mathrm{MHz}$ phase.

As explained above, the WR uses the PPS signal once to reset the counter and lock to a positive-going zero crossing of the $10 \mathrm{MHz}$ from the Cs frequency output. This lock of the WR is supposed to be the first $10 \mathrm{MHz}$ rising edge after the $1 \mathrm{PPS}$ input [10]. A sufficient change in the difference between the phase of the $1 \mathrm{PPS}$ and the $10 \mathrm{MHz}$ would cause additional offsets of $100 \mathrm{~ns}$, upon a power reset. The impact of the phase difference between these two reference signals was verified by adding additional lengths of cable to the 1 PPS cable providing the signal input to the WR GM, followed by cycling the power. The measured offset changed to 175 ns after adding a 43 ns delay, hence causing the WR GM to lock to the subsequent cycle.

The 1 PPS signal into the WR GM is delayed relative to the $10 \mathrm{MHz}$ phase by the 2 additional distribution amplifiers and relative cable delays, as illustrated in Fig. 1. For the arrival time at the WR GM, after the power-cycle, the first positive zero-crossing of the 10 $\mathrm{MHz}$ following the EL DA PPS was measured to be $44 \mathrm{~ns}$. Unfortunately, this measurement was not done before the power cycle. Accounting for the 10 ns EL DA PPS cable delay, the reference zero-crossing that the WR GM is expected to lock to is 54 ns later than the reference plane of the EL DA. The PPS out of the WR GM was measured with equal delay cables to be 88 ns later than the EL DA reference plane before the CCF power cycle, and 75 ns after the power cycle. If we assume the delay from the Cs reference between its PPS and $10 \mathrm{MHz}$ phase was unchanged with the power cycle, there appears to have been a transfer delay in the WR GM of 34 ns before the power cycle, and 21 ns after. On the other hand, it may be that the difference in the output time of the Cs 1 PPS relative to the $10 \mathrm{MHz}$ phase changed by this amount. Since that Cs is an operational part of the NIST NTP server in the 
CCF, it cannot be turned off and on except in an emergency. Additional measurements of similar Cs standards will be reported in later work.

\subsection{White Rabbit Slave Characterization}

The WR Slave device co-located with the testbed reference lab was locked to the WR GM in the CCF, using the White Rabbit protocol [5], as illustrated in Fig. 1. The WR Slave was repeatedly calibrated against the EL DA using the Rb portable clock, in the same manner as the GPS receivers were calibrated, as discussed in section 2.2. Table 2. White Rabbit slave calibration against the EL DA. shows the mean and standard deviation of the Rb runs of the WR Slave against the EL DA. Recall that the WR GM offset from the EL DA changed from 88 ns to 75 ns over the power cycle in the computing facilities. The Rb calibrations of the WR slave device in the testbed reflect the change.

We conservatively estimate a peak-to-peak variation, i.e. a stability estimate, of the WR Slave output as under 2 ns. This stability estimate is based on both the White Rabbit loopback measurements in Fig. 10, and the results of the traveling Rb calibrations in Table 2. However, though the stability is significantly better, because accuracy depends on the Rb calibrations, we are limited in our accuracy uncertainty estimate by the uncertainty of those calibrations. Hence, we estimate an uncertainty of the 1 PPS from the WR Slave against the EL DA and the NTP DA on the order of the Rb uncertainty, namely under 5 ns.

With these estimates, the system can be established as a reference for real-time monitoring of GPS receivers. This is illustrated in Fig. 8 showing two days of data.

Table 2. White Rabbit slave calibration against the EL DA.

\begin{tabular}{lcccc}
\hline $\begin{array}{l}\text { EL DA- } \\
\text { WR Slave }\end{array}$ & $\boldsymbol{\mu}(\mathbf{n s})$ & $\boldsymbol{\sigma}(\mathbf{n s})$ & $\boldsymbol{\mu}(\mathbf{n s})$ & $\sigma(\mathbf{n s})$ \\
\hline Run 1 & 89 & 1.5 & 75 & 2 \\
Run 2 & 90 & 2.5 & 76 & 1.5 \\
Run 3 & 88 & 4 & 76 & 1.5 \\
Run 4 & 92 & 2.5 & 75 & 1.5 \\
Run 5 & 87 & 3 & 74 & 1.5 \\
Run 6 & 86 & 1.5 & 74 & 1.5 \\
Run 7 & 87 & 2.5 & & \\
Run 8 & 89 & 4.5 & & \\
Run 9 & 93 & 2 & & \\
Run 10 & 88 & 1.5 & & \\
\hline Weighted Average & $\mathbf{8 8 . 7}$ & & $\mathbf{7 5 . 0}$ & \\
\hline
\end{tabular}

\subsection{PTP deployment}

Fig. 11 shows the planned network topology of the testbed through which the characterized GPS reference time will be multicasted via PTP Sync messages by at most two time-aware network switches, configured as Transparent Clocks (TCs) to minimize the delay and delay variation. The testbed is comprised of several modules, where each module will have a 
dedicated time-aware network switch to connect to the testbed's experimental measurement and control equipment. This topology determined our testing of the latency of the TC devices that will be used between the PTP GM and any slave devices in the testbed. For Intelligent Electronic Devices (IEDs), the devices will be configured to use the peer-to-peer delay mechanism via the Ethernet transport layer based on the IEEE C37.238 PTP Power Profile. The benefit of the peer-to-peer delay mechanism is that each link of the network trajectory computes the link delay and residence time and therefore the delay is known by the slave upon receipt of the synchronization packet and is used to adjust the time offset (3). The delay is computed per link using the peer delay request and peer delay response messages to compute delay forward. However, the restriction to achieve the required synchronization is that all links must support PTP.

$$
\text { offset }=t_{1}+\text { delay }_{\text {forward }},
$$

For general CPS and IoT case studies where devices may go through additional switches and routers without on path timing support, the end-to-end delay mechanism will be used. The end-to-end delay mechanism does not need full on-path timing support, but requires four timestamps from PTP event or follow-up messages to adjust the time offset (4): the origin timestamp from the GM $\left(t_{1}\right)$, the time the synchronization message arrives at the slave $\left(t_{2}\right)$, the time the slave sends a delay request message $\left(t_{3}\right)$ and the time the GM receives the delay request message $\left(t_{4}\right)$.

$$
\text { offset }=\frac{\left(t_{2}-t_{1}\right)+\left(t_{3}-t_{4}\right)}{2},
$$

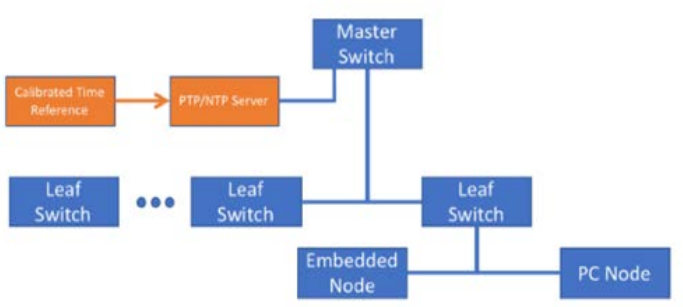

Fig. 11. Smart Grid CPS testbed network.

\section{Timing Characterization Methodology and Results}

\subsection{GPS Reference Calibrations}

The GPS receivers in the testbed were all intended to be configured to produce UTC(USNO) via GPS as stationary receivers with fixed coordinates. We must note, however, that GPS2 was found at times to not be in fixed position mode, but rather was continuing to estimate its position.

As discussed above, receiver calibration is necessary because of an unknown constant bias in each of the receivers due to delays in the antenna, antenna cable and receiver. Since the value of UTC(USNO) from GPS was consistently within 5 ns of UTC(NIST), we used the measurements of UTC(NIST) via the NTP reference to calibrate the receiver offsets. UTC(NIST) is estimated at the Gaithersburg, MD NTP reference using common-view time transfer to NIST, Boulder, CO [4]. 
As mentioned previously, the calibration of GPS receivers in the testbed against UTC via the CCF receiver changed by roughly 100 ns given the \pm 10 ns uncertainty, coincident with a planned power outage of the CCF lab containing the NTP UTC reference, the weekend of April 13, 2018. There is no known reason for any of these receivers to shift due to a power cycle.

Obelow gives some of the calibrations against UTC via the NTP reference. The GPS receivers are labeled as GPS1-4. The uncertainty in these calibrations is dominated by the 10 ns uncertainty due to the short measurements of the GPS receivers during the round-trip Rb calibrations. One of the factors contributing to the mean receiver offsets $(\mu)$ listed in Table 3 is the delay in the cable connecting the GPS antenna to the GPS receiver. This cable delay is non-trivial since the GPS receiver is located in the basement of a building while the antenna is located on the roof.

Two different cable types were used to connect GPS antennas to the GPS receivers listed in Table 3. GPS 1, 3 and 4 used a Times Microwave LMR 240 cable which is a double shielded $50 \Omega$ cable. GPS 2 used a Belden 9104 (RG-59 variant) cable, which is a double shielded 75 $\Omega$ cable. Measurements were performed to determine the electrical propagation delay introduced by both cables as shown in Table 4 . Both time domain and frequency domain methods were adopted as independent verification of the cable delay. The tests described here were replicated for both cables used with appropriate modifications to the measurement fixture to ensure impedance matching. 
Table 3. Testbed GPS receiver offsets from UTC(NIST) via Rb calibrations against the NTP reference receiver.

\begin{tabular}{|c|c|c|c|c|}
\hline \multirow[b]{2}{*}{$\begin{array}{l}\text { UTC- } \\
\text { GPS }\end{array}$} & \multicolumn{2}{|c|}{ Pre-Power Cycle } & \multicolumn{2}{|c|}{ Post-Power Cycle } \\
\hline & $\mu(n s)$ & $\sigma(n s)$ & $\mu(n s)$ & $\sigma(n s)$ \\
\hline GPS1 & 418 & 9 & 527 & 12 \\
\hline GPS2 & 359 & 4 & 452 & 15 \\
\hline GPS3 & 390 & 12 & 492 & 9 \\
\hline GPS4 & N/A & N/A & 539.7 & 8.5 \\
\hline
\end{tabular}

Time domain measurements were performed using standard reflectometry techniques. The setup consisted of an impulse generator connected to an impedance matched power splitter, the splitter output was connected to the long coaxial cable being tested with the other end of the cable being left open. The time delay of the primary reflected mode of the incident impulse induced by the impedance discontinuity at the open end of the cable was used to measure the electrical propagation delay. A time domain filter or gate was used to suppress unwanted signal components such as multi-reflections and harmonics. A two-step approach was used to ensure a reliable measurement free of bias errors in the reflected mode. First, a 100 ns wide pulse with a steep rising edge was used to tune the gate filter to admit a single reflection. Second, a cardinal sine function $[\operatorname{sinc}(\mathrm{x})$ ] centered at $1.5 \mathrm{MHz}$ was used to determine the appropriate trigger level for the reflected mode. Note, that the sinc function transformed to the frequency domain has an ideal "rectangular" frequency response. The band limited nature of this impulse function reduces the impact of aliased components on the amplitude of the reflected mode.

The gated data was independently evaluated in the frequency domain using the $\mathrm{S}_{11}$ parameter representation. Here a frequency sweep was applied spanning the L1 C/A GPS bandwidth 1575.42 MHz $\pm 1.023 \mathrm{MHz}$ and the linear phase lag along a Voltage Standing Wave Ratio (VSWR) circle for the injected frequency sweep was used to determine a best fit approximation of the transmission line delay. Measurements obtained for both cables using both techniques is presented in Table IV. We also include mechanical length of the cable estimated using manufacturer specifications for each cable. Note the good correspondence in measured electrical delay between both measurement techniques.

Table 3 shows the large change of order 100 ns in receiver offsets against the UTC value provided via the NTP reference from before to after the power outage in the CCF. This then becomes the uncertainty of our UTC estimates. To resolve this, we sent the receiver GPS2 to NIST Boulder to calibrate it directly against UTC(NIST). The calibration was done using the antenna for GPS2 from Gaithersburg. But there was a calibration with each of two different cables in Boulder, both of a similar length to that in Gaithersburg, but the first was a 50 Ohm cable, which did not match the specified impedance of the receiver and antenna, while the second was a matching 75 Ohm cable. In both calibrations, the measured delay of the receiver and antenna combined was zero within the uncertainty, after removing the measured calibration antenna cable delay. The combined uncertainty was $16 \mathrm{~ns}$, almost entirely due to the instability in the receiver GPS2. That is, any delay in the receiver and antenna appears to be removed, perhaps because the manufacturer has accounted for them in firmware. Hence the results of the NIST, Boulder, calibrations would predict that the UTC time offset in 
Gaithersburg would be simply the value of the antenna cable delay measurements in Table 4. Thus the UTC time offset of GPS2 in Gaithersburg is approximately $378 \mathrm{~ns}$, with a combined uncertainty of 16 ns. This appears to agree with the value measured before the power outage. While this increases confidence in our measurements, we await a new receiver and another independent calibration of that one, before we can consider reducing our uncertainty.

Table 4. GPS Antenna Cable Measurements

\begin{tabular}{lcc}
\hline & RG 59 & LMR 240 \\
\hline Time delay & $378 \mathrm{~ns}$ & $374 \mathrm{~ns}$ \\
Nominal delay & $3.9 \mathrm{~ns} / \mathrm{m}$ & $3.9 \mathrm{~ns} / \mathrm{m}$ \\
Cable length estimate & $96.0 \mathrm{~m}$ & $94.2 \mathrm{~m}$ \\
Frequency delay & $377 \mathrm{~ns}$ & $374 \mathrm{~ns}$ \\
Cable length estimate & $95.4 \mathrm{~m}$ & $94.5 \mathrm{~m}$ \\
\hline
\end{tabular}

\subsection{PTP characterization}

For the PTP network characterization, to measure the synchronization offset through the TCs we explored the use of the test setup as shown in Fig. 12. Measurements were the PPS outputs from the reference and slave clocks using a Time Interval Counter with $100 \mathrm{ps}$ calibrated accuracy. The PTP emulator device used both a $10 \mathrm{MHz}$ and 1 PPS input reference from the testbed's WR slave clock. The test device emulated the Grandmaster and a slave. In addition, there is an actual slave on the network with a PPS output. Since all devices have onpath PTP support, we configured the GM, TCs, and slave to use the two-step clock and peerto-peer delay mechanism communicating on layer 2 Ethernet protocol as required in the PTP Power Profile standards for substation automation [12][13]. The end-to-end synchronization uncertainty was also based on the network topology in Fig. 12.

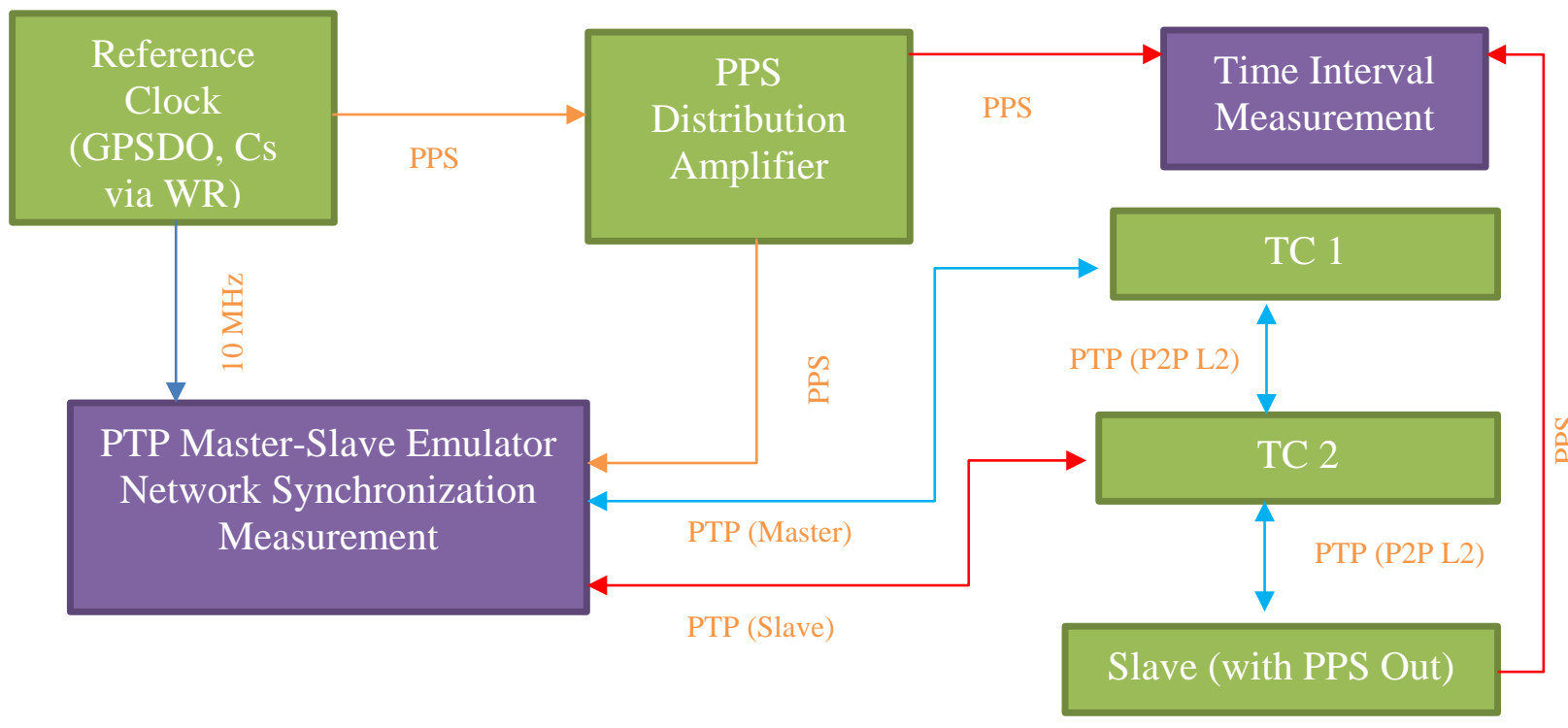

Fig. 12. Transparent clock characterization topology. The lines in red denote the measured signals or packets. The offsets are measured by the time interval counter and the time stamp and packet delays are measured by the network synchronization tester. 
Table 5. Network Device Time Uncertainty Contribution Using PTP Peer-to-Peer Layer 2

\begin{tabular}{|c|c|c|c|c|}
\hline \multirow[b]{2}{*}{ Number of TCs } & \multicolumn{4}{|c|}{ WR Clock Reference - PTP Slave Offset } \\
\hline & $\mu(n s)$ & $\sigma(n s)$ & $\min (n s)$ & $\max (n s)$ \\
\hline 1 & 15 & 5 & -5 & 33 \\
\hline 2 & 17 & 6 & -2 & 36 \\
\hline 3 & 19 & 7 & -1 & 40 \\
\hline $\begin{array}{c}\text { Uncertainty } \\
99.7 \% \text { confidence }\end{array}$ & & ns $(3 \sigma$ & & \\
\hline
\end{tabular}

Based on the PTP devices and measurement capability available, Table 5 shows the testbed was capable of achieving a peak-to-peak offset of -2 to $36 \mathrm{~ns}$ for 2 TCs using peer-to-peer layer 2 protocol, where the master and slave are configured to use two-step clocks, with follow-up messages. This models what we expect for many nodes on the testbed. 

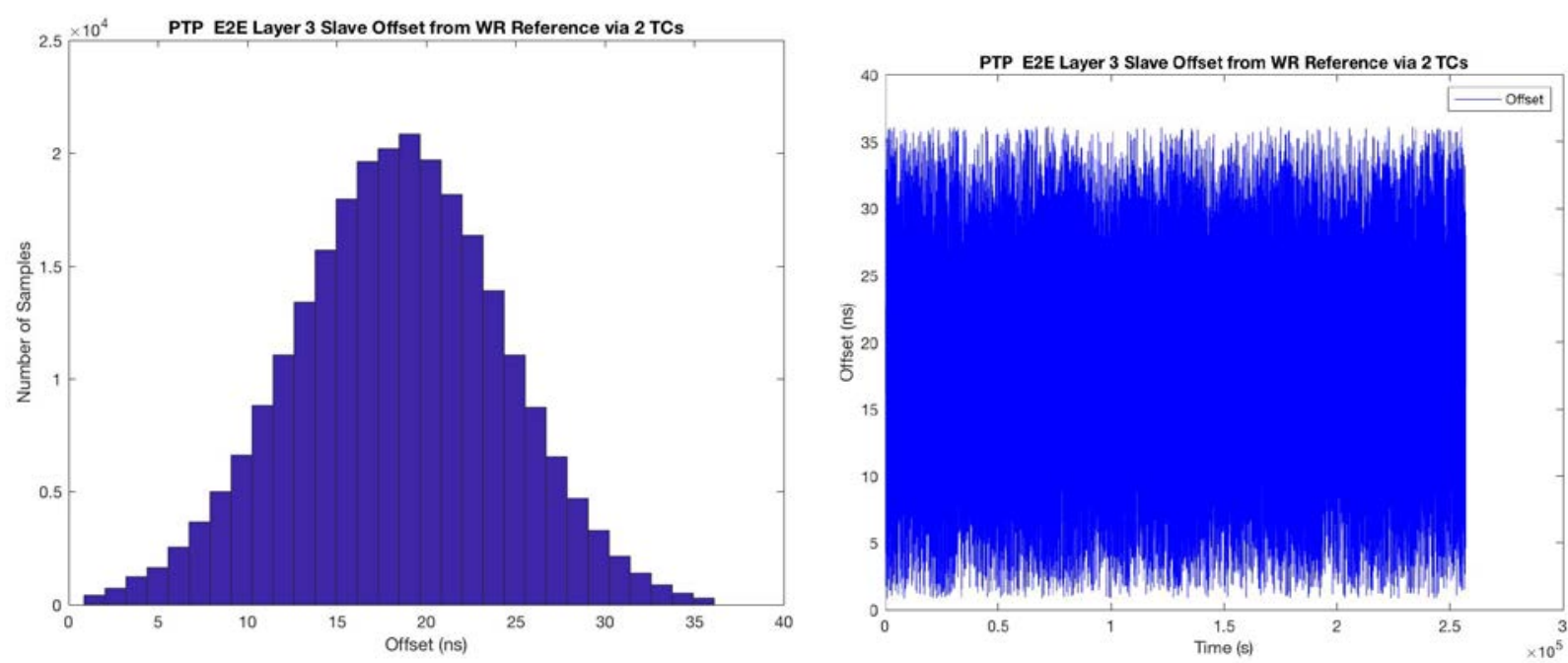

Fig. 13. Time offset distribution between the Cs reference source (via the WR network) and the PTP slave via 2 TCs configured as PTP P2P Layer 2.

The Lilliefors goodness-of-fit test was used to verify the normality of the distribution, shown in Fig. 13. Time offset distribution between the Cs reference source (via the WR network) and the PTP slave via 2 TCs configured as PTP P2P Layer 2., with a p-value of less than 0.001. Applying the central limit theorem, the mean and 3 sigma standard deviation was used to derive the uncertainty of the empirical measurements. At $99.7 \%$ confidence interval the time uncertainty contribution of the 2 TCs is on the order of $18 \mathrm{~ns}$ for the experiment given a measurement interval of over $3600 \mathrm{~s}$.

The other topology of interest in the testbed is end-to-end layer 3 for application layer communications. End-to-end can also be used in environments where there are intermediary switches and end devices without hardware PTP support in large scale CPS and IoT networks. The tests were limited to only switches with PTP support to serve as a baseline for future characterization without full on-path PTP support. The results are shown in Table 6 with an uncertainty of 15 ns. The normality of the distribution, shown in Fig. 14 was verified with a p-value equal to 0.001. Fig. 15 shows the TDEV comparison [14] of the different reference sources and time distribution methods available in the testbed.

Table 6. Network Device Time Uncertainty Contribution Using PTP End-to-End Layer 3

\begin{tabular}{|c|c|c|c|c|}
\hline \multirow[b]{2}{*}{ Number of TCs } & \multicolumn{4}{|c|}{ WR Clock Reference - PTP Slave Offset } \\
\hline & $\mu(n s)$ & $\sigma(n s)$ & $\min (n s)$ & $\max (n s)$ \\
\hline 1 & 24 & 4 & 13 & 35 \\
\hline 2 & 25 & 4 & 12 & 39 \\
\hline 3 & 28 & 5 & 14 & 42 \\
\hline $\begin{array}{c}\text { Uncertainty } \\
99.7 \% \text { confidence }\end{array}$ & & 5 ns (3c & & \\
\hline
\end{tabular}



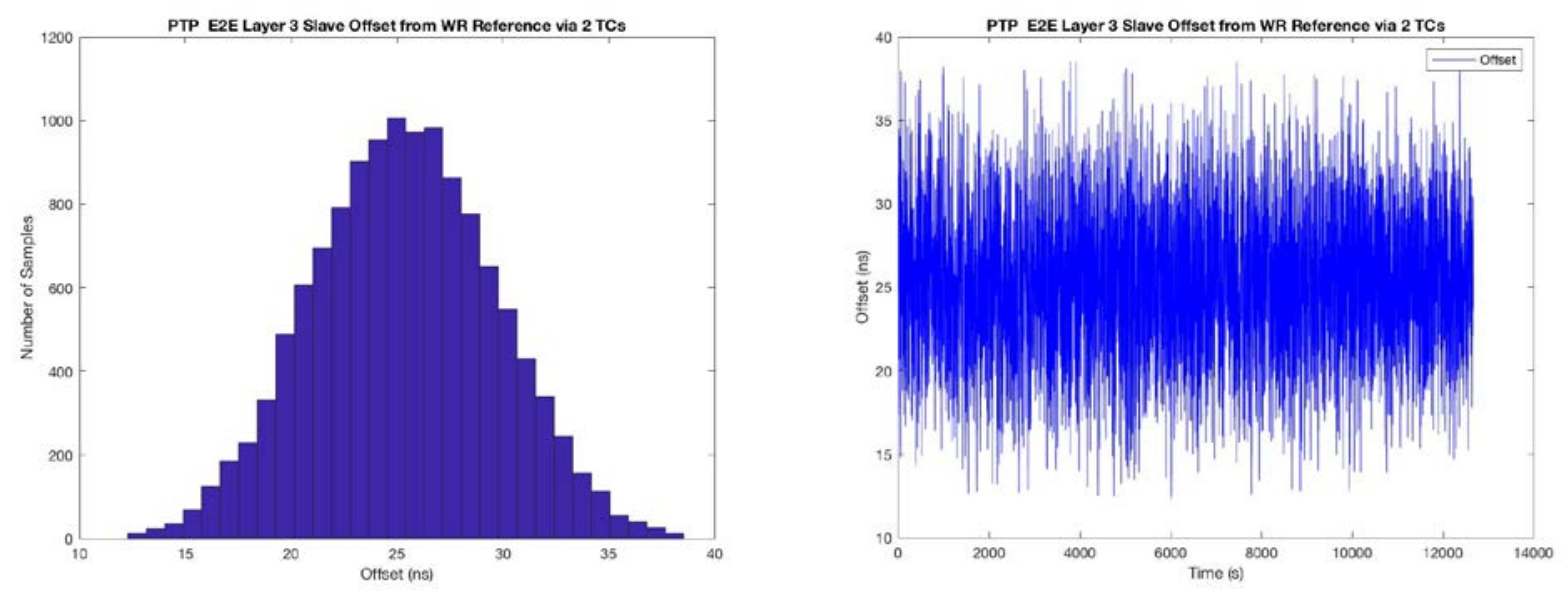

Fig. 14. Time offset distribution between the Cs reference source (via the WR network) and the PTP slave via 2 TCs configured as PTP E2E Layer 3 with a PTP GM emulation.

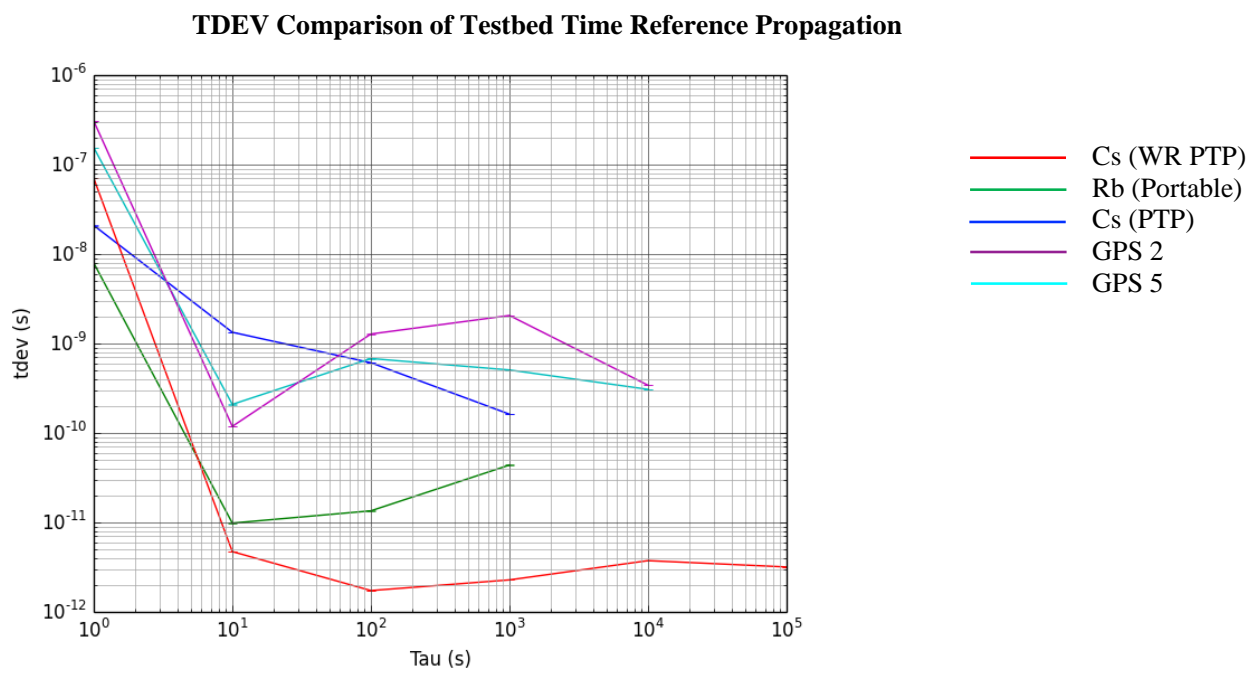

Fig. 15. TDEV comparison of testbed time reference distribution methods.

\subsection{End node characterization}

One of the purposes of calibrating the GPS receiver is to enable traceable characterization and monitoring of end node timing performance. Following the analysis in the previous section having the use of a PTP slave with hardware timing support, this section evaluates the software-based time synchronization. Metrics of interest include (1) the system clock offset relative to the master and (2) the timestamping error of physical input signals. All experiments on the embedded device were performed in headless mode.

To test the clock synchronization achievable on an embedded computing system in the PTP network, the PTP daemon (PTPd) [15] service was installed on the embedded device running Linux as a PTP end-to-end layer 3 slave that will synchronize its system clock with a local grandmaster clock, as shown in Fig 16. The node goes through a PTP TC and a single switch 
without on-path support. Through monitoring the log files produced by PTPd, we obtained the device's system clock offset, mean path delay, and clock correction in parts per million (ppm) relative to the grandmaster clock after the arrival of every Sync message. The mean time offset error was approximately $50 \mu \mathrm{s} \pm 10 \mu \mathrm{s}$.

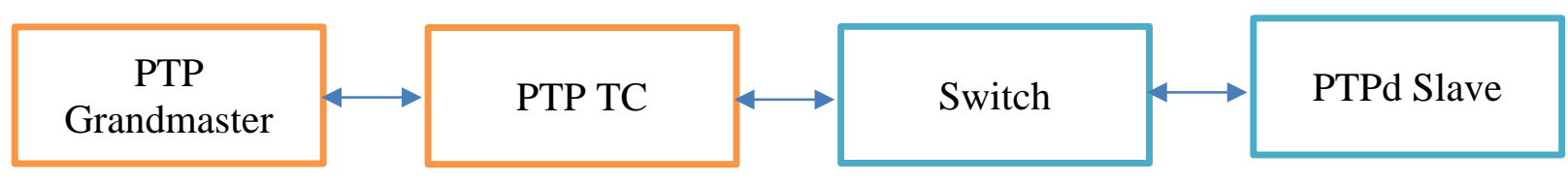

Fig 16. Test topology with software-based PTP timestamping and a non-PTP switch.

To test the timestamping error of an input signal, an embedded computer's system clock was synchronized to the NIST NTP [16][17][18] servers. To measure the general-purpose input output (GPIO) timestamping error of a physical signal, an application was developed on the embedded computing device to timestamp the top of the second marker of the $100 \mathrm{~Hz}$ Interrange Instrumentation Group (IRIG)-B signal using the device's system clock, as shown in Fig. 17. The measured error is the difference between the decoded IRIG-B time and the system timestamp. Based on test observation intervals of 12 hours, the aggregate timestamp error was on the order of $8 \mathrm{~ms} \pm 500 \mu \mathrm{s}$. The sources of the delays and delay variations can be attributed to the computational delay to decode the signal, the GPS receiver errors, the asymmetry of the networks delays between the embedded node and the NTP server, system time-stamping latency. Future efforts will focus on improving the methodology or code efficiency and disaggregating the sources of error to determine the individual contributions.

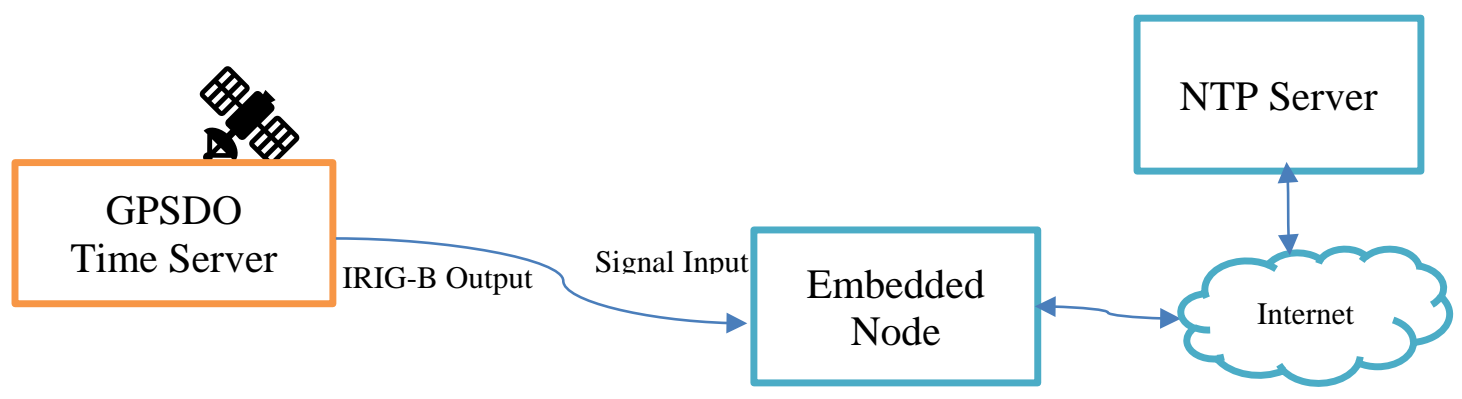

Fig. 17. Test setup for estimating the aggregate timestamp error of a 'typical' IoT node synchronized to an NTP server.

\section{Conclusion}

Time is considered an integral element of CPS [19]. Synchronized clocks enable the continued advancement of distributed measurement, communications and control technologies. Equally important is the ability to measure the delay characteristics of network of clocks in order to monitor the clock and respective timestamp errors. Understanding the clock stability and factors impacting clock frequency errors enables the ability to predict time 
and timestamp errors, which can provide useful contextual information on the clock and timestamp quality on time-dependent measurements and other applications when a reference source is unavailable in a CPS environment. To compensate for prediction errors based on imperfect models and whims of the physical world, another solution is online monitoring of the distributed system[20][21]. This technical note documents the initial effort to build the test infrastructure and explore test methodologies for online monitoring of time errors, while striving to base the methodologies upon the foundational elements of time and frequency metrology[4][6][22][23].

We have shown how, using a combination of methods, to calibrate the timing of nodes in a testbed. This timing includes relative time against a local reference plane with an uncertainty of $5 \mathrm{~ns}$, and the uncertainty of UTC against that reference plane of $16 \mathrm{~ns}$. Nevertheless, the cycling of power in systems appears to have changed measurements against UTC by 100 ns, which therefore must be our final uncertainty, since this remains unexplained. An independent receiver calibration has improved the confidence in our measurements, but we are waiting for another new receiver to be calibrated to reduce our uncertainty. Local measurements of the White Rabbit system changed by 13 ns. These issues underscore the importance of repeated calibrations, online monitoring, and the use of multiple UTC references to ensure calibrations stay within requirements.

\section{Acknowledgments}

We greatly appreciate the support provided by Dr. Judah Levine, the NIST Central Computing Facilities team for hosting the equipment, the NIST Networking team for oversight on the cable installations, Stefania Romisch and Bijunath Patla of NIST Boulder for a receiver calibration, and the support of the NIST Smart Grid Cyber-Physical Systems Program, including David Wollman, Chris Greer, and the Software Systems Division including Eric Simmon and John Messina.

\section{References}

[1] International reference time scales, BIPM website: https://www.bipm.org/en/bipmservices/timescales/.

[2] NIST Time Frequently Asked Questions (FAQ), NIST website: https://www.nist.gov/pml/time-and-frequency-division/nist-time-frequently-askedquestions-faq.

[3] “Time Synchronization in the Electric Power System.” NASPI Technical Report, March 2017.

https://www.naspi.org/sites/default/files/reference_documents/tstf_electric_power_syste m_report_pnnl_26331_march_2017_0.pdf.

[4] D.W. Allen and M.A. Weiss (1980). Accurate Time and Frequency Transfer During Common View, (May), Proc. 34 th Annual Symp. On Frequency Control, pp 334-346, 1980, available from: https://tf.nist.gov/general/pdf/192.pdf.

[5] M. Lipinski et al. (2012). "Performance results of the first white rabbit installation for CNGS time transfer" 2012 International IEEE Symposium on Precision Clock Synchronization for Measurement Control and Communication (ISPCS) 24-28 September 2012, pp 1-6. 
[6] J. Levine, 1999. Introduction to time and frequency metrology. Review of scientific instruments, 70(6), pp.2567-2596.

[7] D.W. Allan, M.A. Weiss, J.L. Jespersen, "A frequency-domain view of time-domain characterization of clocks and time and frequency distribution systems," Proc. $45^{\text {th }}$ Annual Symp. On Frequency Control, 1991.

[8] D. A. Howe, T. K. Peppler, "Definitions of Total Estimators of Common Time-Domain Variances,” Proc. 2001 IEEE Intl. Frequency Control Symp., pp.127-132, 2001, available from https://tf.nist.gov/general/pdf/1481.pdf .

[9] M.A. Weiss, V. Zhang, W. Lewandowski, P. Uhrich, D. Valat, "NIST and OP GPS receiver calibrations spanning twenty years: 1983 - 2003,” Proc. European Frequncy and Time Forum, 2004, available online from http://tf.nist.gov/general/pdf/1946.pdf .

[10] Z. Jiang, D. Matsakis, and V. Zhang, “Long-term instability in UTC time links,” Proc. 2017 Precise Time and Time Interval Meeting, pp. 105-126, 2017, available online from http://tf.nist.gov/general/pdf/2902.pdf .

[11] T. Wlostowski. Note on using the WR Switch in Grandmaster mode, 08.10.2012. Frequently asked questions about the White Rabbit Switch. https://www.ohwr.org/projects/white-rabbit/wiki/faqswitch, accessed June 27, 2018. https://doi.org/10.1049/cje.2015.04.031

[12] IEC/IEEE International Standard - Communication networks and systems for power utility automation Part 9-3: Precision time protocol profile for power utility automation," in IEC/IEEE 61850-9-3 Edition 1.0 2016-05 , vol., no., pp.1-18, 31 May 2016.

[13] IEEE Standard Profile for Use of IEEE 1588 Precision Time Protocol in Power System Applications," in IEEE Std C37.238-2017 (Revision of IEEE Std C37.238-2011) , pp.142, 19 June 2017.

[14] AllanTools API Documentation, https://allantools.readthedocs.io/en/latest/api.html, March 2018.

[15] K. Correll, N. Barendt, and M. Branicky, 2005, October. Design considerations for software only implementations of the IEEE 1588 precision time protocol. In Conference on IEEE Precision Clock Synchronization, pp. 11-15.

[16] D.L. Mills, 1991. Internet time synchronization: the network time protocol. IEEE Transactions on communications, 39(10), pp.1482-1493.

[17] D.L. Mills, 2003. A brief history of NTP time: Memoirs of an Internet timekeeper. ACM SIGCOMM Computer Communication Review, 33(2), pp.9-21.

[18] J. Levine, M.A. Lombardi, and A.N.Novick, 2002. NIST computer time services: Internet Time Service (ITS), Automated Computer Time Service (ACTS), and time. gov web sites. NIST Special Publication, 250, p.59.

[19] Griffor, E.R., Greer, C., Wollman, D.A. and Burns, M.J., 2017. Framework for CyberPhysical Systems: Volume 1, Overview (No. Special Publication (NIST SP)-1500-201).

[20] J.V. Deshmukh, A. Donzé, S. Ghosh, X. Jin, G. Juniwal and S.A. Seshia, 2017. Robust online monitoring of signal temporal logic. Formal Methods in System Design, 51(1), pp.5-30.

[21] M. Mehrabian,, M. Khayatian, A. Mousa, A. Shrivastava, Y.S. Li-Baboud, P. Derler , E. Griffor, H.A. Andrade, M. Weiss, J.C. Eidson, and D. Anand, 2018, June. An efficient timestamp-based monitoring approach to test timing constraints of cyber-physical systems. In Proceedings of the 55th Annual Design Automation Conference (p. 144). 
[22] C. Hackman, and D.B. Sullivan, 1995. Resource Letter: TFM-1: Time and frequency measurement. American Journal of Physics, 63(4), pp.306-317.

[23] S. Bregni, 1997. Clock stability characterization and measurement in telecommunications. IEEE Transactions on instrumentation and measurement, 46(6), pp.1284-1294. 